\title{
Interventional changes of thyroid hormone levels do not affect circulating irisin levels in humans
}

Grigorios Panagiotou, $^{1,2}$, Kalliopi Pazaitou-Panayiotou ${ }^{2}$, Despina Komninou ${ }^{2,3}$, Stavroula A. Paschou1,4, Nikolaos Kalogeris4, Andromachi Vryonidou', Christos S. Mantzoros ${ }^{1}$

${ }^{1}$ Division of Endocrinology, Diabetes and Metabolism, Department of Internal Medicine, Beth Israel Deaconess Medical Center, Harvard Medical School, Boston, MA, USA

Department of Endocrinology- Endocrine Oncology, Theagenio Cancer Hospital, Thessaloniki, Greece

3 Clinical Nutrition Laboratory, Department of Nutrition/Dietetics, Alexander Technological Education Institute of Thessaloniki

4Department of Endocrinology and Diabetes, Hellenic Red Cross Hospital, Athens, Greece

\section{INTRODUCTION}

Thyroid hormones and irisin increase energy expenditure and induce browning of adipose tissue. However, little is known about irisin physiology and regulation. Here we aim to elucidate whether thyroid hormones alter circulating irisin concentrations in humans.

\section{DESIGN AND STUDIES}

One cross-sectional (case-control) evaluation and two interventions were performed independently from each other, including previously thyroidectomized patients.

- Case-control study : Ninety-six subjects under thyroxine treatment, were recruited to study any associations between anthropometric parameters, thyroid axis hormones and irisin and then divided into a "euthyroid" and a "subclinical hyperthyroid" group, based on their Thyroid Stimulating Hormone levels (TSH cut-off value of $0.3 \mathrm{mIU} / \mathrm{L}$ ).

- Interventional Study A: Thirty-four patients that were followed-up for thyroid cancer, were studied during a 5-week withdrawal from L-T4 treatment, according to a standardized routine protocol for preparation for whole body scan and/or thyroglobulin measurement. Blood samples were drawn at baseline and at the end of the study period.

- Interventional Study B: Thirteen patients underwent a recombinant human TSH (rhTSH) stimulation protocol and without receiving radioactive iodine for whole body scan and blood samples were drawn at baseline, day 3 (i.e. at least 24 hours after the second intramuscular injection), day 5 and day 10 .

\section{RESULTS}

- Serum irisin levels were not cross-sectionally associated with anthropometric parameters as well as TSH, free thyroxine and free triidothyronine, in either the whole cohort or in the euthyroid and/or subclinical hyperthyroid subgroups.

- There was no significant difference between euthyroid and subclinical hyperthyroid subjects $(p=0.60)$.

- L-T4 withdrawal did not alter irisin levels ( $p=0.33$ ) (FIGURE A).

- The effect of rhTSH stimulation on circulating irisin was also null $(p=0.60)$ (FIGURE B).

\section{FIGURES}

A.

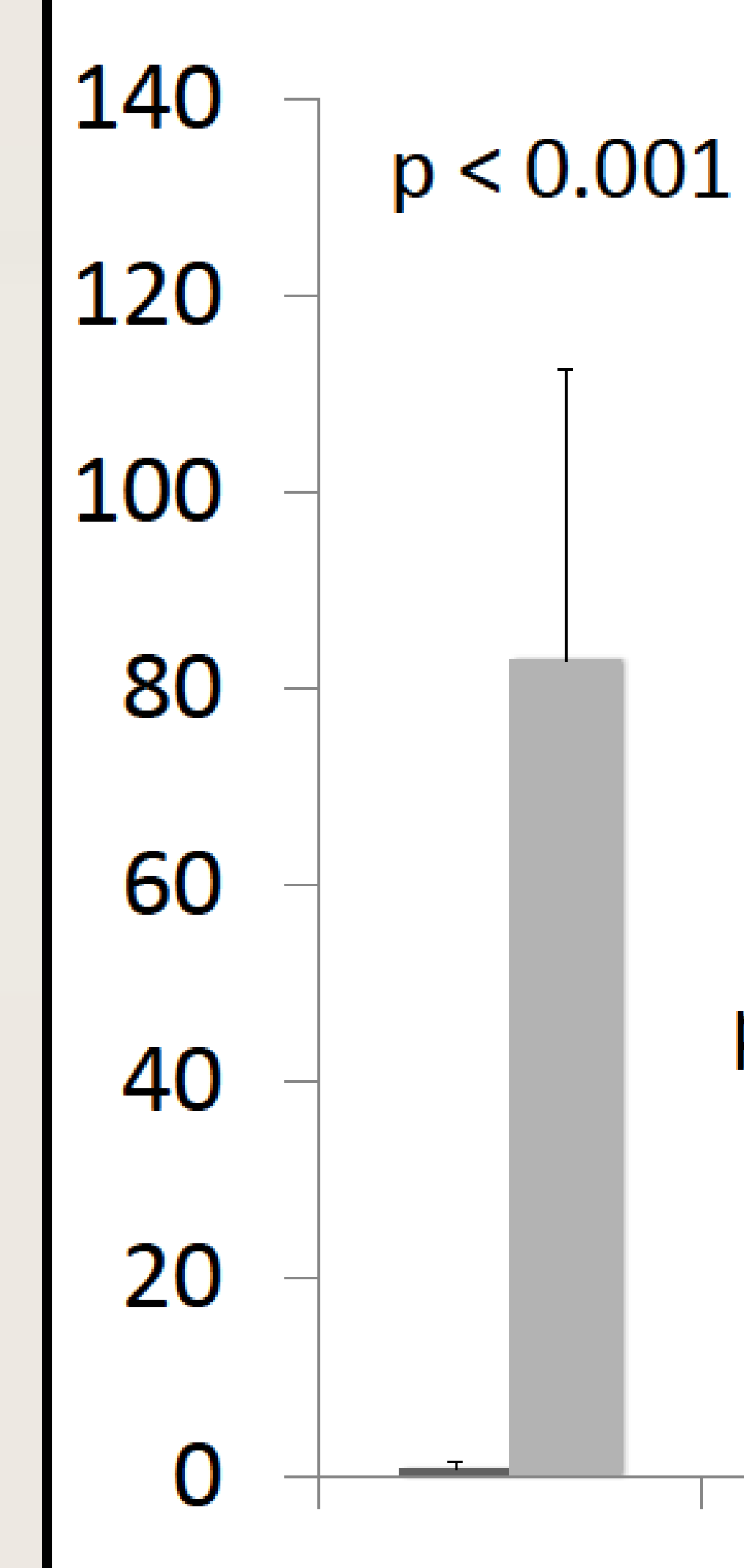

TSH Free T4 Total T3 Irisin $(\mathrm{mlU} / \mathrm{L})(\mathrm{pg} / \mathrm{mL})(\mathrm{nmol} / \mathrm{L})(\mathrm{ng} / \mathrm{mL})$ $\square$ OFF THYROXINE

\section{B.}

270

240

210

180

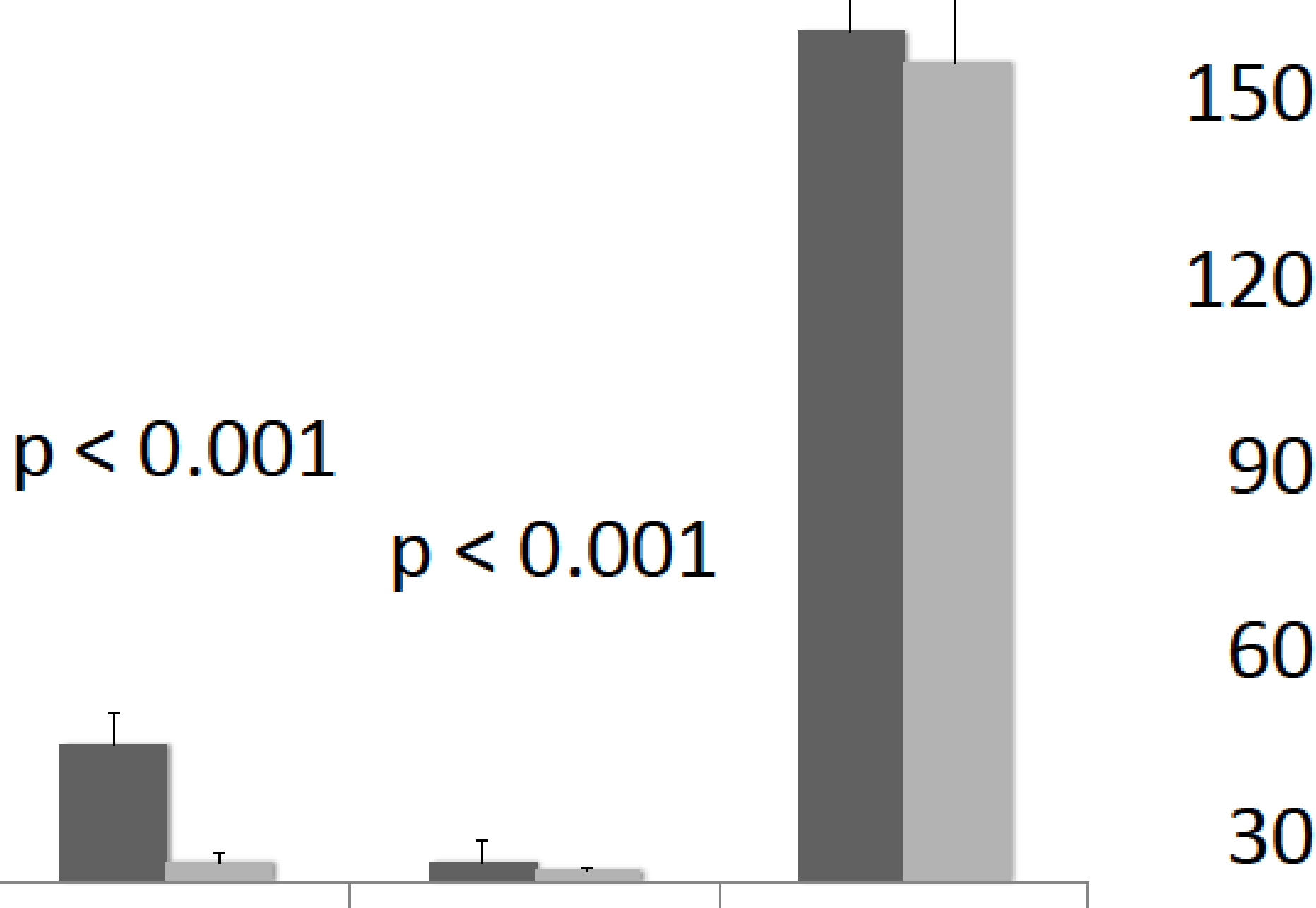

30

0

Day 1 Day 3 Day 5 Day 10

\section{CONCLUSIONS}

Changes of thyroid hormone levels within the physiological or mildly supraphysiological range do not affect circulating irisin levels in humans. Therefore, irisin's proposed metabolic effects are most likely independent from thyroid axis hormones.

For more information, please contact

Grigorios Panagiotou, MD, email: gpanayio@gmail.com

Christos S. Mantzoros, MD, DSc, PhD he mult, email: cmantzor@bidmc.harvard.edu 